\title{
Development and Ex Vivo Characterization of Enteric Coated Chitosan Beads for Crohn's Disease Management
}

Craig Mendonca

University of Connecticut, craig.mendonca@uconn.edu

Follow this and additional works at: https://opencommons.uconn.edu/usp_projects

Part of the Cellular and Molecular Physiology Commons, Medicinal and Pharmaceutical Chemistry Commons, Other Pharmacy and Pharmaceutical Sciences Commons, and the Pharmaceutical Preparations Commons

\section{Recommended Citation}

Mendonca, Craig, "Development and Ex Vivo Characterization of Enteric Coated Chitosan Beads for Crohn's Disease Management" (2018). University Scholar Projects. 56.

https://opencommons.uconn.edu/usp_projects/56 
Development and Ex Vivo Characterization of Enteric Coated Chitosan Beads for Crohn's

Disease Management

Craig Mendonca

Thesis advisors: Dr. Diane Burgess, Dr. Mary Bruno, Dr. Akiko Nishiyama

Table of Contents

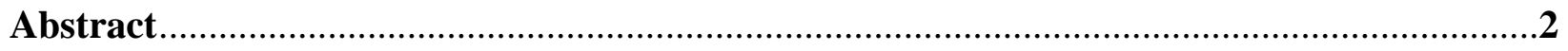

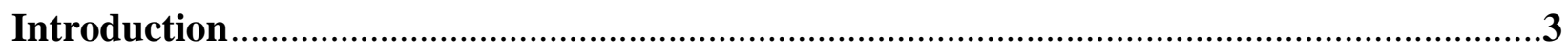

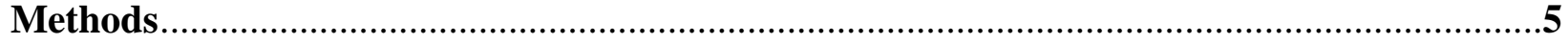

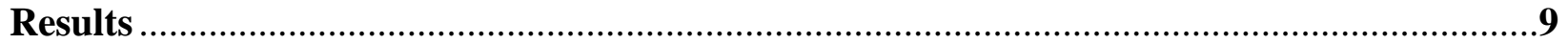

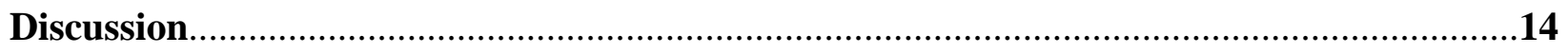

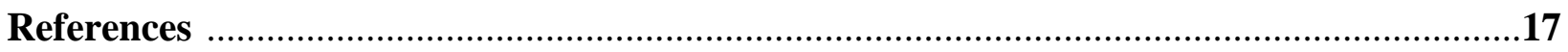




\begin{abstract}
:
Drug delivery is the process of transporting a drug to its site of action. It includes controlled delivery, where the drug is released at the tissue in a regulated manner. Additionally, it includes targeted delivery, where the drug is aimed at a specific tissue or region to avoid unwanted side effects (that would occur if the drug were distributed throughout the body). A cellulose acetate phthalate coated chitosan bead was developed as a formulation for controlled and targeted release of dexamethasone to the ilium and colon. This formulation was determined to be effective in delaying dexamethasone release until the formulation had reached conditions that simulated the large intestine. Ex vivo characterization using an adapted Franz diffusion cell demonstrated that the formulation was able to limit the diffusion of the drug through the intestine, which may reduce unwanted side effects as result of the dexamethasone entering the systemic circulation.
\end{abstract}




\section{Introduction:}

Patients with Crohn's Disease present with incurable inflammation of the ileum and colon. This results in symptoms such as abdominal pain, chronic diarrhea, weight loss, and rectal bleeding [1], which can be treated by anti-inflammatory drugs targeted to the intestines. Several methods exist to facilitate this aim: 1) Prodrugs that are inert in the small intestine but are activated by the microbiota enzymes; 2) $\mathrm{pH}$ sensitive polymers that are used to coat the drug and then degrade at a target $\mathrm{pH}$ range in the GI tract; and 3) bacterial degradable polymers or matrix/hydrogel systems that are broken down by microbiota enzymes to release drug [2]. A bead formulation using $\mathrm{pH}$ sensitive polymers could, therefore, potentially be a method to deliver an antiinflammatory drug and control its release in a temporal and spatial manner. Chitosan is a common polymer that has been used previously for colonic drug delivery, due to its selective degradation in the colon. Furthermore, chitosan has been shown to be safe for patients with Crohn's disease and does not exacerbate symptoms [3]. An oral formulation would have to traverse the GI tract in order to reach the inflamed areas. Gastric juice presents a complication for many drugs and delivery methods as the acid tends to dissolve polymers and damage molecules. Cellulose acetate phthalate (CAP) is a polymer that is insoluble in the acidic gastric environment, making it a good candidate for the enteric coating that will surround the chitosan beads. At the intestinal $\mathrm{pH}$ of 6.8 , the polymer is soluble, allowing dissolution over the course of the small intestine [4]. A previous polymer formulation used CAP with a modified chitosan (crosslinked with polyethylene glycol) for chemotherapy targeting of the colon [5]. This study demonstrates that CAP and chitosan are compatible and may be useful for colon targeting of other drugs. Dexamethasone (DEX) is a corticosteroid that acts as an anti-inflammatory agent; such drugs are often used as treatments for Crohn's disease [6]. As an immunosuppressant drug, 
DEX reduces inflammation by inhibiting leukocyte infiltration of the affected area [7]. However, IV, IM, or oral corticosteroids can have musculoskeletal side effects such as metabolic bone disease, osteoporosis, osteopenia, osteomalacia, and osteonecrosis. Additionally, the risk of sepsis can be an issue, since DEX will inhibit immune system function outside the targeted region [6]. The CAP coating would allow the beads to safely pass through the stomach and the upper intestinal region intact but wear off throughout the rest of the intestines to release DEX from the chitosan beads. Accordingly, this would release the drug in the colonic region, provide local action at the disease site, and avoid unwanted systemic side effects. Therefore, this CAP and chitosan formulation should delay DEX release until the region where the anti-inflammatory drug is most needed. 


\section{Methods:}

Solutions: The 3\% (w/v) chitosan solution was prepared by adding $1.2 \mathrm{~g}$ of chitosan to $40 \mathrm{ml}$ of $\mathrm{dH}_{2} \mathrm{O}$ and $0.8 \mathrm{ml}$ glacial acetic acid. This solution was stirred for 16 hours at $70^{\circ} \mathrm{C}$. The $5 \%$ (w/v) tripolyphosphate (TPP) solution consisted of $10 \mathrm{~g}$ TPP and $200 \mathrm{ml}$ of $\mathrm{dH}_{2} \mathrm{O}$, with the $\mathrm{pH}$ adjusted to 6 . The $5 \%$ CAP solution was prepared by vortexing $2.5 \mathrm{~g}$ of CAP with $47.5 \mathrm{ml}$ of acetone and $2.5 \mathrm{ml}$ of a saturated methylene blue solution in acetone. Artificial gastric acid was prepared by adding $2.9 \mathrm{ml}$ of $37 \% \mathrm{HCl}$ to $500 \mathrm{ml}$ of $\mathrm{dH}_{2} \mathrm{O}$; the $\mathrm{pH}$ was then adjusted to 1.5 . Artificial intestinal fluid was prepared by adding hydrochloric acid to a $\mathrm{pH} 7.4$ phosphate buffer saline (PBS) solution until the $\mathrm{pH}$ reached 6.8. The ethanol DEX solution was made by adding $40 \mathrm{mg}$ of micronized DEX to $2 \mathrm{ml}$ of HPLC grade ethanol. This solution was vortexed for 5 minutes and sonicated for 15 minutes to form a cloudy saturated solution. The PBS DEX solution (for FDC) was made by adding $2.5 \mathrm{mg}$ of micronized DEX to $50 \mathrm{ml}$ of $\mathrm{pH} 7.4 \mathrm{PBS}$. This was vortexed for 5 minutes and sonicated for 15 minutes until all powder was dissolved. The mobile phase for HPLC was 60\% Millipore filtered water, 39.5\% HPLC grade acetonitrile $(\mathrm{ACN})$, and $0.5 \%$ of a $10 \%$ phosphoric acid solution $(\mathrm{v} / \mathrm{v})$. This was mixed and then sonicated for 30 minutes to remove dissolved gas.

Bead preparation: Chitosan beads were formed by dropping 3\% (w/v) chitosan at a rate of 0.8 $\mathrm{ml} / \mathrm{min}$ into a stirring $5 \%(\mathrm{w} / \mathrm{v})$ TPP solution. Beads were dropped at a height of $4 \mathrm{~cm}$ from the surface of the liquid and stirred at $300 \mathrm{rpm}$. This method, adapted from Shiraishi et al., induced crosslinking of the chitosan polymer, and created a porous bead. After 1 hour, the TPP was diluted from the stirring solution and the beads were freeze dried for 48 hours at $-20^{\circ} \mathrm{C}$. The dried beads were soaked in a $20 \mathrm{mg} / \mathrm{ml}$ DEX solution for 1 hour to allow DEX to penetrate the beads. The solvent was evaporated and the beads were washed three times with $100 \%$ ethanol. 
The DEX loaded beads were sprayed in 10 second pulses by ultrasonic atomization of a $5 \%$ (w/v) CAP solution at $130 \mathrm{KHz}$, as described by Ganguly et al. This solution also contained methylene blue dissolved in acetone, which was used to visualize the CAP layer on the chitosan beads. Beads were rotated while the solvent evaporated in a vacuum between pulses to ensure an even coating around all sides of the beads. The beads were vacuum dried one final time to remove all of the solvent.

In vitro drug release: In vitro release was tested using an adapted USP-1 method [9]. Equal masses of the CAP-coated and noncoated (NC) formulation were placed into seperate USP-1 baskets. The baskets were submerged in separate $50 \mathrm{ml}$ conical centrifuge tubes that were prefilled with $25 \mathrm{ml}$ of artificial gastric acid. $100 \mu \mathrm{l}$ samples were taken from the sampling port in the basket at each time point. The centrifuge tubes were incubated in a $37^{\circ} \mathrm{C}$ shaking water bath at $100 \mathrm{rpm}$ for the duration of the experiment. After 1 hour, the baskets were removed from the tubes and dried. The baskets were then submerged in $25 \mathrm{ml}$ of the artificial intestinal fluid and returned to the same water bath for the remaining time points. Samples were diluted with the mobile phase and filtered with a $22 \mu \mathrm{m}$ filter prior to High Performance Liquid Chromatography (HPLC) analysis.

Drug loading: A spatula was used to crush and powder each of the formulations. Aliquots of the $\mathrm{NC}$ and CAP formulation were mixed with $30 \mathrm{ml}$ of $50 \%$ methanol and sealed in an Erlenmeyer flask. These were sonicated in a water bath for 1.5 hours until the formulation was dissolved. The flasks were stirred prior to filtering $1 \mathrm{ml}$ of the solution through a $22 \mu \mathrm{m}$ filter for HPLC analysis.

HPLC method: Samples were analyzed using an Agilent® Zorbax SB-C18 column (5 $\mu$ m, 4.6 $\mathrm{mm} \times 15 \mathrm{~cm}$ ). Prior to usage, the column was equilibrated with the mobile phase for 1 hour at a 
flow rate of $1 \mathrm{ml} / \mathrm{min} .20 \mu \mathrm{l}$ of each sample was injected and ran for 6 minutes with detection at $240 \mathrm{~nm}$. The average retention time for DEX was 4.6 minutes. This peak was integrated and a standard curve was used to determine the concentration of DEX in the sample. After all of the runs had been completed, the column was washed for 45 minutes with $100 \%$ ACN.

Rat intestine preparation: Rats were fasted for 1 day prior to sacrifice. The large intestine was harvested from the cecum to the rectum and suspended in sterile Mammalian Ringer's solution at $4^{\circ} \mathrm{C}$. Intestine segments were flushed with cold $\mathrm{pH}$ 7.4 PBS using a blunt end syringe to remove any remaining fecal matter. The intestine was incised along the edge of the taeniae coli to remove any fat and open the intestine. Each section was cut into squares with a side length of 8 mm and were kept submerged in cold PBS until the Franz diffusion cells were ready. Franz diffusion cell: PermeGear® Franz diffusion cells with a $15 \mathrm{~mm}$ orifice diameter were fitted with a Teflon washer adaptor to decrease the orifice area. The inner diameter of the adaptor was $5 \mathrm{~mm}$ and it was $0.5 \mathrm{~mm}$ thick. The receptor chamber was filled with $12 \mathrm{ml}$ of $\mathrm{pH} 7.4 \mathrm{PBS}$, which was stirred at $600 \mathrm{rpm}$ for the duration of the experiment. The cells were allowed to equilibrate at $37^{\circ} \mathrm{C}$ for 1 hour prior addition of the tissue segments. One rat intestine square was sandwiched between two adaptors with the lumen facing the donor chamber; this assembly was secured with a pinch clamp. $10 \mathrm{mg}$ of the powdered chitosan formulation was added to $10 \mathrm{ml}$ of $\mathrm{pH}$ 7.4 PBS and stirred at $400 \mathrm{rpm}$. Immediately, $1 \mathrm{ml}$ of this suspension or $1 \mathrm{ml}$ of the PBS DEX solution was added to the donor chamber and the top was sealed. A dispensing needle was used to remove a $0.3 \mathrm{~mL}$ sample from the sampling port at each time point and sample volume was replaced with fresh $\mathrm{pH}$ 7.4 PBS. Each sample was run through a $22 \mu \mathrm{m}$ filter prior to HPLC. 

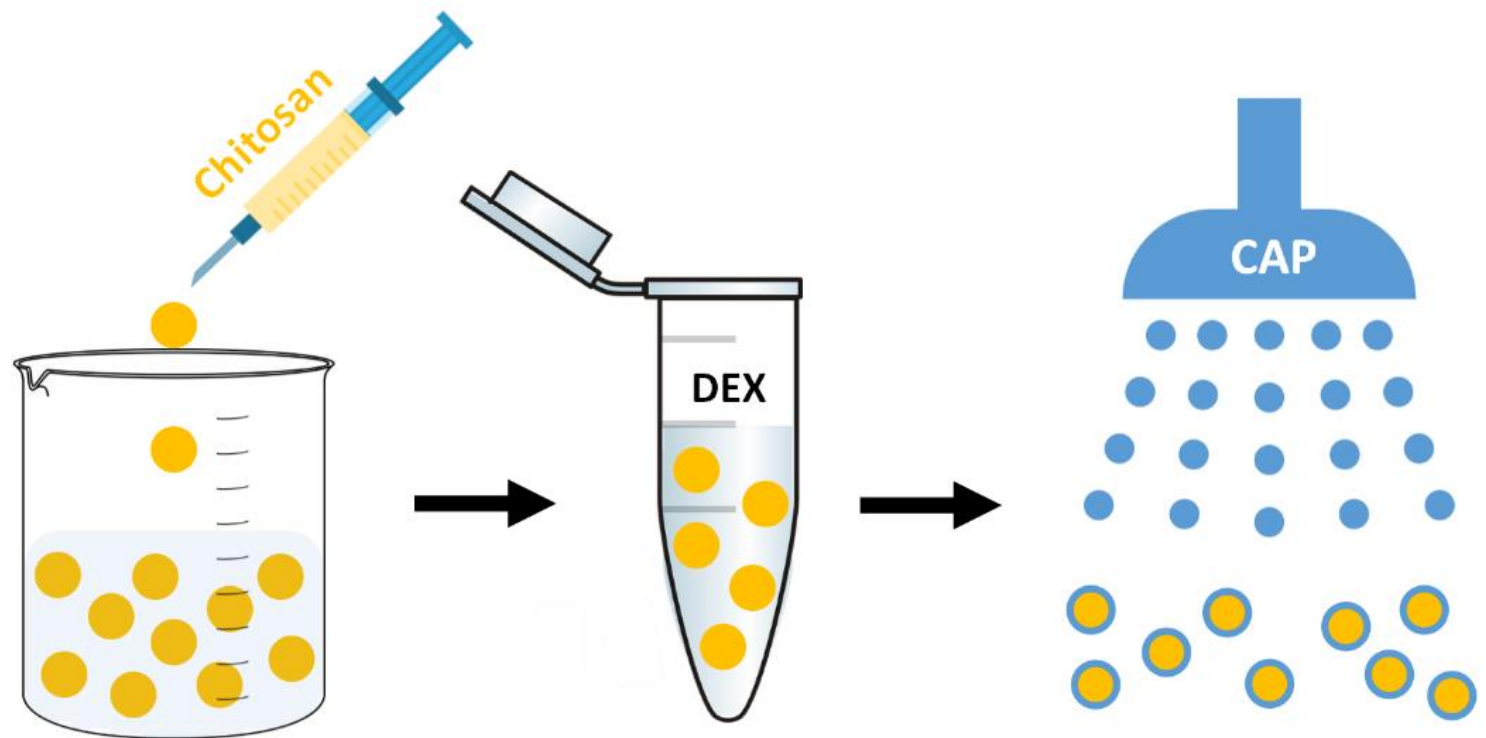

Figure 1: Schematic representation of the method of chitosan bead preparation. Chitosan was dropped into the stirring TPP solution to create beads, then soaked in a DEX solution. Finally, the DEX loaded beads were coated with CAP via pulses of ultrasonic atomization. 


\section{Results:}

Rounded CAP coated chitosan beads were

constructed as described in Figure 1. DEX was

loaded into the beads and the percent of DEX per

mass of the beads was determined (Table 1). The

greater drug loading for the noncoated beads can

be attributed to the additional mass added to the

formulation during the ultrasonic atomization.

Since the CAP was stained with methylene blue,

the CAP coated beads appeared visibly blue, as

seen in Figure 2.

The drug release and disintegration of the CAP

coated and noncoated beads were examined in

artificial gastric acid for one hour, and then the

beads were transferred to a $\mathrm{pH} 6.8$ PBS solution

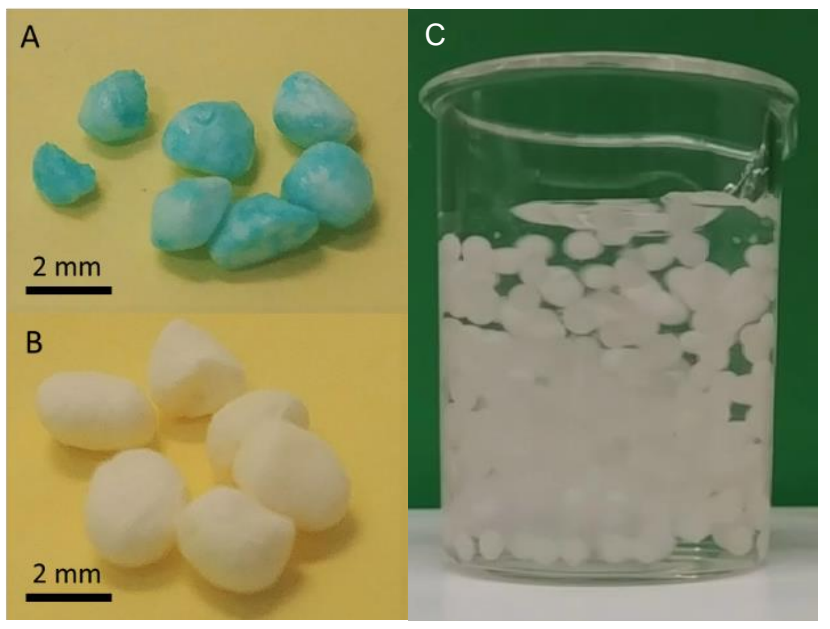

Figure 2: Chitosan beads loaded with DEX, CAP coated (A) or noncoated (B). The CAP beads were stained with methylene blue. Before coating, the beads were crosslinked in a stirring TPP solution (C).

\begin{tabular}{|c|c|}
\hline & Drug Loading \% (w/w) \\
\hline CAP & $4.545 \pm 0.244$ \\
\hline NC & $5.298 \pm 0.212$ \\
\hline
\end{tabular}

Table 1: Drug loading percent (w/w) of DEX inside the CAP coated beads and noncoated beads.

that simulated the intestinal environment. Within 30 minutes, most of the noncoated beads had dissolved into the solution (Figure 3) since chitosan is a hydrophilic polymer that is soluble in acid. The CAP coated beads maintained their structural integrity throughout the artificial gastric acid condition. CAP is a white polymer, so methylene blue was added to the CAP solution before it was sprayed onto the chitosan beads. Consequently, the thickness and coverage of the coating could be assessed. The blue CAP coating remained on the beads while in the artificial gastric acid, but dissolved in the artificial intestinal fluid (Figure 3). After the CAP coating had worn away, the beads began to follow the same disintegration pattern as the noncoated beads in gastric 
acid, but it was much slower since the $\mathrm{pH}$ of the artificial intestinal fluid is closer to neutral. The drug release profile was consistent with these observations (Figure 4). The noncoated beads showed an immediate release of DEX, with more than $75 \%$ of the total drug released within the first half hour in gastric juice. The majority of the drug had already been released before this formulation was transferred to the artificial intestinal fluid. The CAP coated beads showed limited DEX release in the artificial gastric juice during the first hour of testing. After they were transferred to the artificial intestinal fluid, the CAP coated beads quickly released the remaining DEX into the neutral $\mathrm{pH}$ medium.

Diffusion of DEX through a section of the large intestine was examined using the Franz diffusion cell apparatus, shown in Figure 6. Since the CAP coating would be worn away in the small intestine, the powdered noncoated chitosan formulation was compared to the PBS DEX solution. The permability data was linearized by plotting the square root of time against the mass of DEX that crossed the colon section per area of tissue. In order to compare the formulation to the solution, the quantities of DEX measured were scaled to match the difference between the drug loading of the formulation and the concentration fo the DEX solution. (Figure 5). The diffusion of DEX through the intestine was two-fold greater for the DEX solution than the chitosan forulation for the first time point. For the last time point, the diffusion of the DEX solution through the intestine was three-fold greater than DEX in the chitosan formulation. Thus there was an increasingly greater release over time from the DEX solution. The slope of the least squares regression line for the DEX solution was 15.66, whereas the slope of the least-squares regression line for the chitosan formlation was 4.873 . This indicates that the rate of diffusion of DEX was faster for the solution than the formulation. 
CAP

$0 \min$

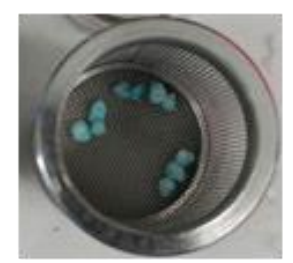

$15 \min$

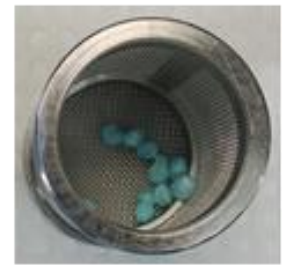

$30 \mathrm{~min}$

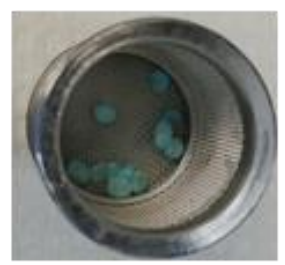

$45 \min$

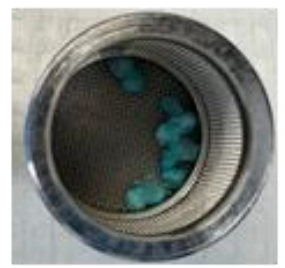

1 hour
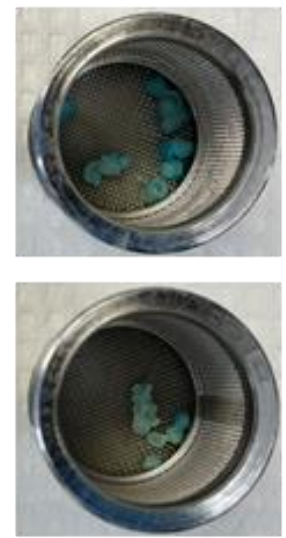

2 hours

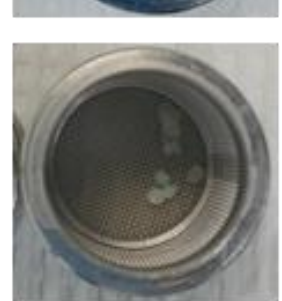

4 hours

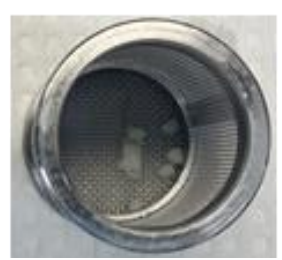

NC
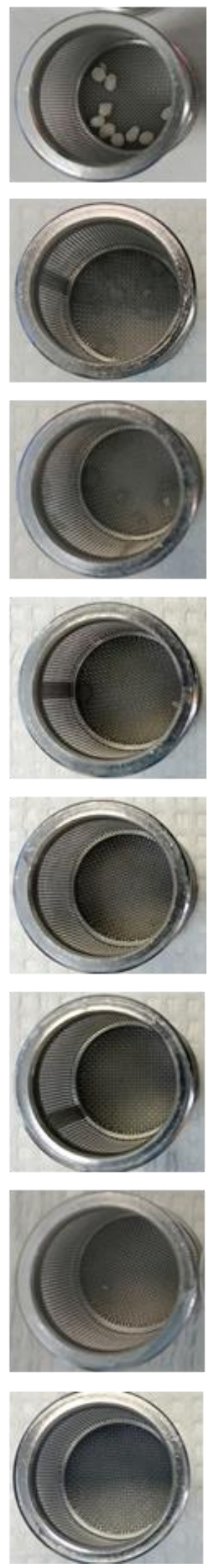

Figure 3: Morphological comparison of CAP coated and noncoated (NC) beads. The beads were incubated at $37^{\circ} \mathrm{C}$ at 100 rpm in artificial gastric acid for one hour and then in the artificial intestine fluid for the remaining time. 


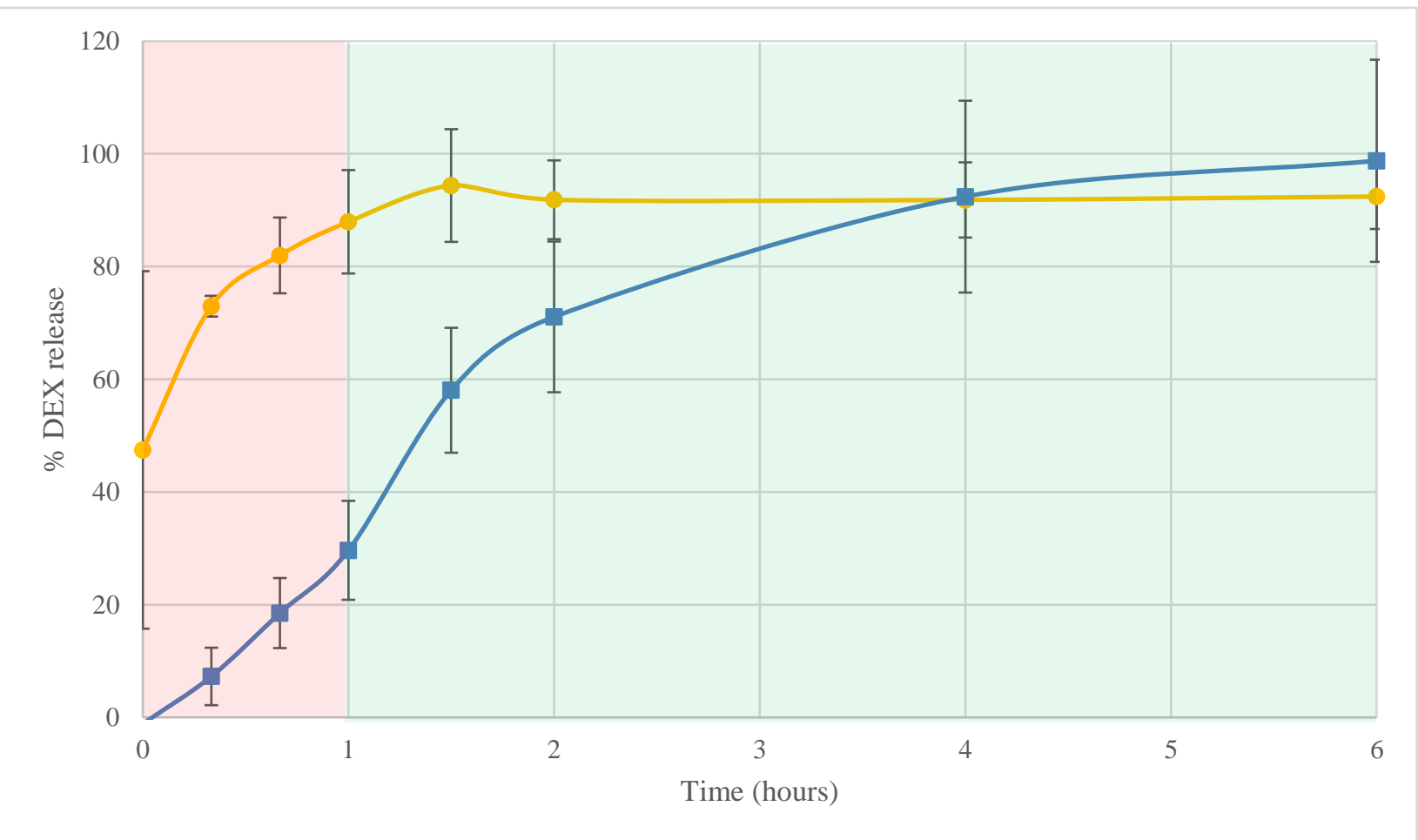

Figure 4: Percent of DEX released at each time point for CAP coated beads (blue square) and noncoated beads (yellow circle). Beads were in artificial gastric acid (red background) for the first hour and then were switched to artificial intestinal fluid (green background) for the remaining time.

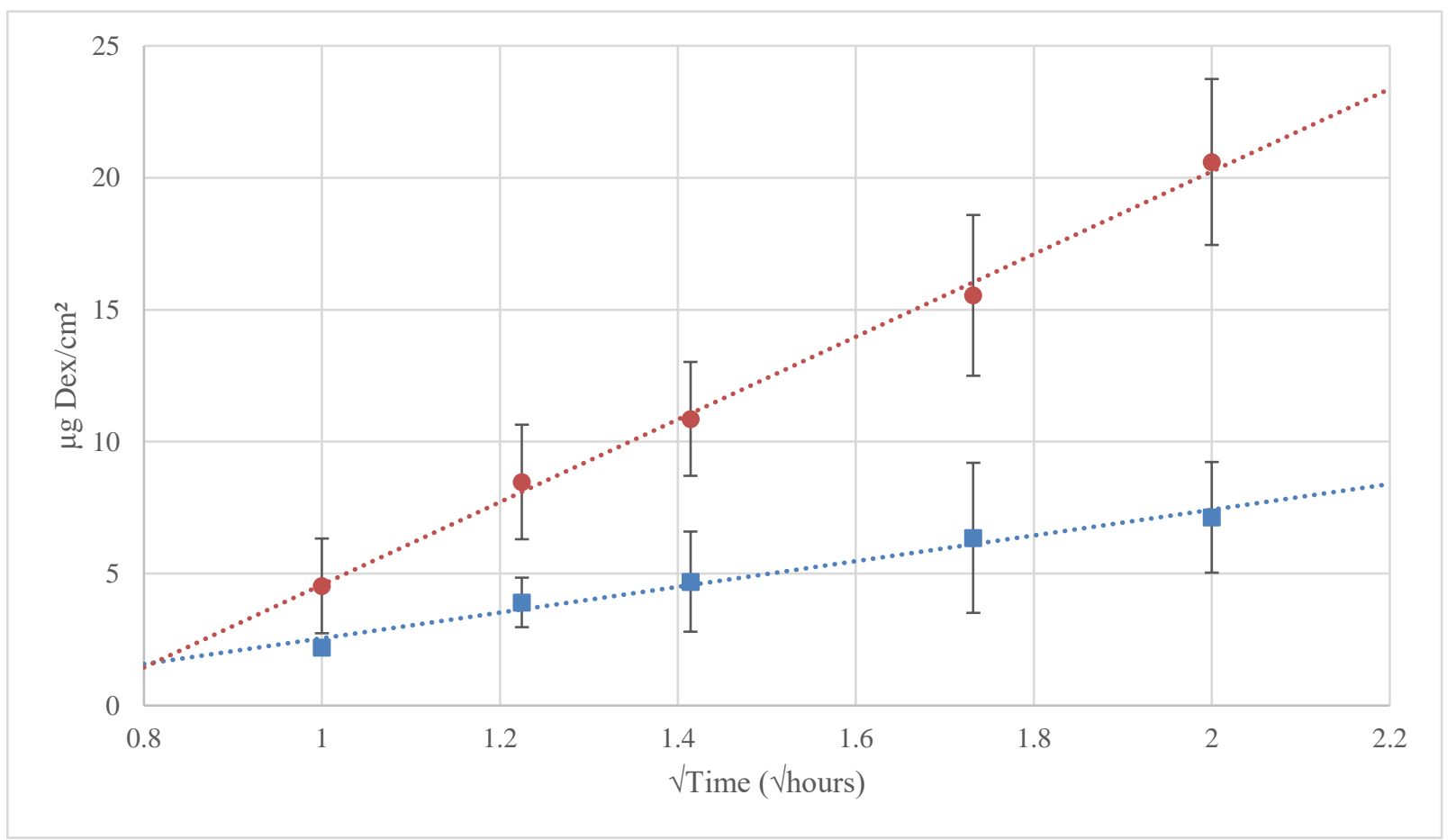

Figure 5: Diffusion of DEX through the large intestine as measured by the Franz diffusion cell. The DEX solution showed a faster release (red circles, $\mathrm{R}^{2}=0.9964$ ) than the chitosan formulation (blue squares, $\mathrm{R}^{2}=0.9775$ ) over four hours. 
Mendonca 13

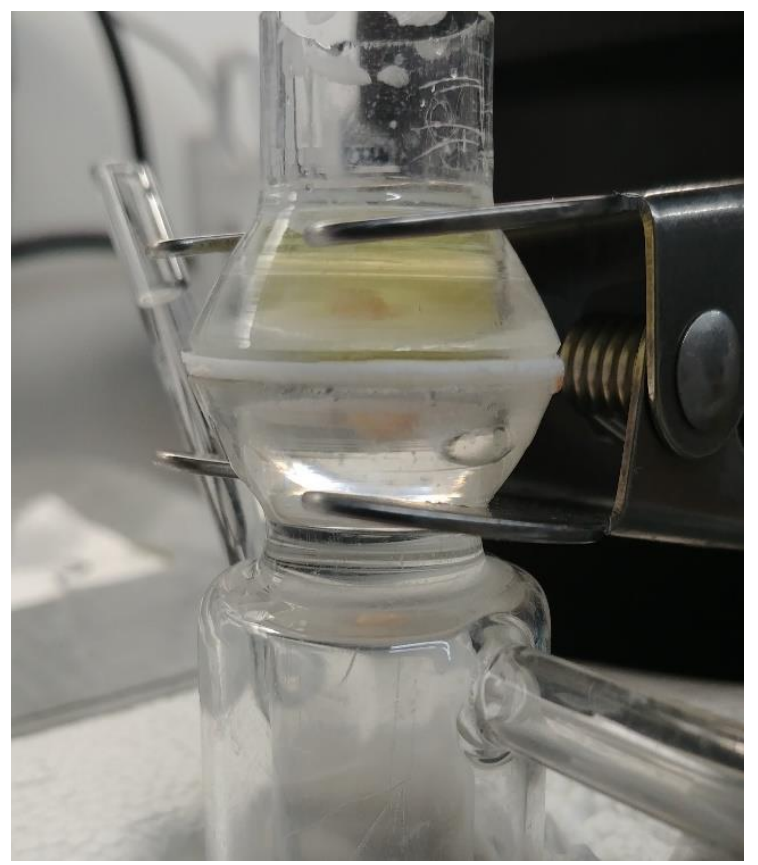

Figure 6: Franz diffusion cell adapted to an orifice diameter of $5 \mathrm{~mm}$. The rat intestine is sandwiched between the white Teflon washers. The yellow solution in the donor chamber (top) does not leak into the receptor chamber (bottom). A water pump was used to maintain a temperature of $37^{\circ} \mathrm{C}$. 


\section{Discussion:}

In this study, an enteric coating made of CAP was developed to cover the surface of a chitosan bead, which was loaded with DEX (Figure 2). The CAP was stained with methylene blue so that the coating thickness could be estimated visually. Furthermore, the status of the coating could be observed in the release media by examining the color change over the course of the experiment. This CAP coating protected the beads from disintegrating in the artificial gastric juice and the CAP coating was subsequently removed in the artificial intestinal fluid. CAP is poorly soluble in an acidic environment, so it remained on the beads. However, it is soluble in a pH neutral environment, so it dissolves into the artificial intestine fluid.

Although the chitosan was protected from dissolving in the artificial gastric acid, about $25 \%$ of the DEX loaded into the beads was still released prior to the $\mathrm{pH}$ change. This indicates that the CAP coating was either not thick enough or still permeable to DEX. The DEX that was released in the stomach would absorb very early in the small intestine. While some forms of Crohn's disease do affect the small intestine, it may be more beneficial to delay the release of the antiinflammatory agent even further than the delay achieved with this method. Nonetheless, the CAP coated beads released the majority of the DEX in the simulated intestinal environment, so this formulation is still superior to the noncoated formulation.

Figure 4 shows that the noncoated formulation released about $90 \%$ of the loaded DEX into the artificial gastric acid. This is consistent with the high solubility of chitosan in an acidic environment. An acetic acid solution was used to dissolve chitosan to make the beads, which has a higher $\mathrm{pH}$ than gastric acid. The chitosan dissolved into the artificial gastric acid, resulting in the DEX being rapidly released as well. This may also explain why the percent release after the basket is switched to the artificial intestine fluid seems to decrease slightly for the noncoated 
formulation. Since the chitosan was fully dissolved in the acid, it was lost when the basket was switched. Consequently, there was no more DEX to be released in those baskets.

The ex vivo testing of the formulation was performed using a rat intestine as the membrane in a Franz diffusion cell. Since the chitosan formulation releases the DEX over the course of several hours, the diffusion rate through the intestine squares was lower than a simple DEX solution in PBS. Since the beads would be moved down the intestine over the course of several hours, a slower diffusion rate may be more advantageous to spread the DEX out over the intestine. The DEX solution may be absorbed too quickly in an earlier region of the intestine. Large amounts of DEX being absorbed would result in the drug entering the systemic circulation, which would increase the potential side effects of this treatment.

The Franz diffusion cell apparatus can be seen in Figure 6. When the rat intestine is sandwiched between the Teflon washers, the tissue tends to sag into the receptor chamber. Intestinal tissue is elastic because of the peristaltic movements required to move digested food and fecal matter through the GI tract. The area of the tissue exposed to the donor chamber is used to calculate the rate that the DEX diffuses through the tissue. This additional curvature would introduce some variability into the area, although it would affect all of the tissue samples similarly. Developing a way to attach the intestine to the Franz diffusion cell adapter to keep it flat may improve the accuracy of the diffusion data. Additionally, DEX may be able to permeate the intestine at different rates depending on the segment of the colon, so examining these separately may yield important information.

In conclusion, an enteric coating made of CAP was developed to cover the surface of a chitosan bead. These beads were loaded with DEX, a strong anti-inflammatory drug. The CAP coating protected the chitosan beads from disintegrating in the artificial gastric juice and the CAP was 
removed in the artificial intestinal fluid. The beads controlled the release of DEX in vitro so that the majority of the drug was released in the basic $\mathrm{pH}$ of the intestines, rather than the acidic $\mathrm{pH}$ of the stomach. Using rat intestine segments in a modified Franz diffusion cell, the ex vivo study shows that the formulation was able to limit the permeation of DEX through the intestine better than the permeation of the PBS DEX solution. These are all characteristics of an ideal Crohn's disease treatment that would help patients manage its unpleasant symptoms. 


\section{References:}

1.) Lichtenstein, G. R., Hanauer, S. B., \& Sandborn, W. J. (2009). Management of Crohn's disease in adults. The American journal of gastroenterology, 104(2), 465-483.

2.) Van den Mooter, G. (2006). Colon drug delivery. Expert opinion on drug delivery, 3(1), 111125.

3.) Tsujikawa, T., Kanauchi, O., Andoh, A., Saotome, T., Sasaki, M., Fujiyama, Y., \& Bamba, T. (2003). Supplement of a chitosan and ascorbic acid mixture for Crohn's disease: a pilot study. Nutrition, 19(2), 137-139.

4.) Maharaj, I., Campbell, J. B., \& Nairn, J. G. (1984). Simple rapid method for the preparation of enteric-coated microspheres. Journal of pharmaceutical sciences, 73(1), 39-42.

5.) Ganguly, K., Aminabhavi, T. M., \& Kulkarni, A. R. (2011). Colon targeting of 5-fluorouracil using polyethylene glycol cross-linked chitosan microspheres enteric coated with cellulose acetate phthalate. Industrial \& Engineering Chemistry Research, 50(21), 11797-11807.

6.) Irving, P. M., Gearry, R. B., Sparrow, M. P., \& Gibson, P. R. (2007). Appropriate use of corticosteroids in Crohn's disease. Alimentary pharmacology \& therapeutics, 26(3), 313 329.

7.) Tsurufuji, S., Kurihara, A., \& Ojima, F. (1984). Mechanisms of anti-inflammatory action of dexamethasone: blockade by hydrocortisone mesylate and actinomycin D of the inhibitory effect of dexamethasone on leukocyte infiltration in inflammatory sites. Journal of Pharmacology and Experimental Therapeutics, 229(1), 237-243.

8.) Shiraishi, S., Imai, T., \& Otagiri, M. (1993). Controlled release of indomethacin by chitosanpolyelectrolyte complex: optimization and in vivo/in vitro evaluation. Journal of controlled release, 25(3), 217-225. 
9.) The United States Pharmacopeia: The National Formulary. (2017). Rockville, MD: The United States Pharmacopeial Convention. 